OPEN $\curvearrowright$ ACCESS

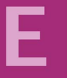
COMMUNICATIONS

ISSN 2056-9890

\section{Crystal structure of 2-(3-nitrophenyl)- 1,3-thiazolo[4,5-b]pyridine}

\author{
Gamal A. El-Hiti, ${ }^{a} *$ Keith Smith, ${ }^{b}$ Amany S. Hegazy, \\ Mansour D. Ajarim ${ }^{c}$ and Benson M. Kariuki ${ }^{\text {b* }}$

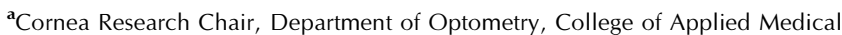 \\ Sciences, King Saud University, PO Box 10219, Riyadh 11433, Saudi Arabia, \\ ${ }^{\mathbf{b}}$ School of Chemistry, Cardiff University, Main Building, Park Place, Cardiff \\ CF10 3AT, Wales, and ${ }^{\mathbf{c} C}$ riminal Evidence, Ministry of Interior, Riyadh 11632, \\ PO Box 86985, Saudi Arabia. *Correspondence e-mail: gelhiti@ksu.edu.sa, \\ kariukib@cardiff.ac.uk
}

Received 9 October 2015; accepted 10 October 2015

Edited by W. T. A. Harrison, University of Aberdeen, Scotland

In the title compound, $\mathrm{C}_{12} \mathrm{H}_{7} \mathrm{~N}_{3} \mathrm{O}_{2} \mathrm{~S}$, the dihedral angle between the planes of the thiazolopyridine ring system (r.m.s. deviation $=0.005 \AA$ ) and the benzene ring is $3.94(6)^{\circ}$. The nitro group is rotated by $7.6(2)^{\circ}$ from its attached ring. In the crystal, extensive aromatic $\pi-\pi$ stacking [shortest centroid-centroid separation $=3.5295$ (9) $\AA$ ] links the molecules into (001) sheets.

Keywords: crystal structure; nitrophenyl; thiazolopyridine derivatives; thiazolo[4,5-b]pyridine.

CCDC reference: 1430578

\section{Related literature}

For a related structure and background references, see: El-Hiti et al. (2015). For further synthetic details, see: Smith et al. (1995); El-Hiti (2003).<smiles>O=[N+]([O-])c1cccc(-c2nc3ncccc3s2)c1</smiles>

\section{Experimental}

\subsection{Crystal data}

$\mathrm{C}_{12} \mathrm{H}_{7} \mathrm{~N}_{3} \mathrm{O}_{2} \mathrm{~S}$

$M_{r}=257.27$

Monoclinic, $P 2_{1} / c$

$a=9.5596(2) \AA$

$b=9.8733(2) \AA$

$c=11.5606(3) \AA$

$\beta=98.122(2)^{\circ}$

$$
\begin{aligned}
& V=1080.20(4) \AA^{3} \\
& Z=4 \\
& \text { Cu K } \alpha \text { radiation } \\
& \mu=2.66 \mathrm{~mm}^{-1} \\
& T=296 \mathrm{~K} \\
& 0.36 \times 0.24 \times 0.03 \mathrm{~mm}
\end{aligned}
$$

\subsection{Data collection}

Agilent SuperNova Dual Source diffractometer with an Atlas detector

Absorption correction: Gaussian (CrysAlis PRO; Agilent, 2014)

$T_{\min }=0.883, T_{\max }=0.986$

\subsection{Refinement}

$R\left[F^{2}>2 \sigma\left(F^{2}\right)\right]=0.031$

$w R\left(F^{2}\right)=0.086$

$S=1.06$

2104 reflections
4063 measured reflections 2104 independent reflections 1930 reflections with $I>2 \sigma(I)$ $R_{\text {int }}=0.016$

Data collection: CrysAlis PRO (Agilent, 2014); cell refinement: CrysAlis PRO; data reduction: CrysAlis PRO; program(s) used to solve structure: SHELXS97 (Sheldrick, 2008); program(s) used to refine structure: SHELXL2013 (Sheldrick, 2015); molecular graphics: ORTEP-3 for Windows (Farrugia, 2012); software used to prepare material for publication: Win GX (Farrugia, 2012) and CHEMDRAW Ultra (Cambridge Soft, 2001).

\title{
Acknowledgements
}

The authors extend their appreciation to the Criminal Evidence Department, Ministry of Interior, Riyadh, Saudi Arabia, for funding this research and to Cardiff University for continued support.

Supporting information for this paper is available from the IUCr electronic archives (Reference: HB7519).

\section{References}

Agilent (2014). CrysAlis PRO. Agilent Technologies, Yarnton, England. Cambridge Soft (2001). CHEMDRAW Ultra. Cambridge Soft Corporation, Cambridge, Massachusetts, USA.

El-Hiti, G. A. (2003). Monatsh. Chem. 134, 837-841.

El-Hiti, G. A., Smith, K., Hegazy, A. S., Ajarim, M. D. \& Kariuki, B. M. (2015). Acta Cryst. E71, o866.

Farrugia, L. J. (2012). J. Appl. Cryst. 45, 849-854.

Sheldrick, G. M. (2008). Acta Cryst. A64, 112-122.

Sheldrick, G. M. (2015). Acta Cryst. C71, 3-8.

Smith, K., Anderson, D. \& Matthews, I. (1995). Sulfur Lett. 18, 79-95. 


\title{
supporting information
}

Acta Cryst. (2015). E71, o877 [https://doi.org/10.1107/S2056989015019118]

\section{Crystal structure of 2-(3-nitrophenyl)-1,3-thiazolo[4,5-b] pyridine}

\author{
Gamal A. El-Hiti, Keith Smith, Amany S. Hegazy, Mansour D. Ajarim and Benson M. Kariuki
}

S1. Introduction

As part of our ongoing studies of thiazolopyridines (El-Hiti et al., 2015), the title compound was prepared by two different processes (El-Hiti, 2003; Smith et al., 1995) and its structure was determined.

\section{S2. Experimental}

\section{S2.1. Synthesis and crystallization}

2-(3-Nitrophenyl)-1,3-thiazolo[4,5-b]pyridine was obtained in 90\% yield from acid hydrolysis (HCl, $5 \mathrm{M})$ of 3-(diisopropylaminothiocarbonylthio)-2-(3-nitrophenylcarbonylamino)pyridine under reflux for $5 \mathrm{~h}$ (Smith et al., 1995) or in $58 \%$ yield from reaction of 3-(diisopropylaminothiocarbonylthio)-2-aminopyridine with 3-nitrobenzoic acid in the presence of phosphorus oxychloride under reflux for $4 \mathrm{~h}$ (El-Hiti, 2003). Crystallization of the crude product from chloroform gave the title compound as colourless crystals. The structure of the title compound was elucidated by various spectroscopic and analytical data, which were consistent with those reported (Smith et al., 1995).

\section{S2.2. Refinement}

$\mathrm{H}$ atoms were positioned geometrically and refined using a riding model with $U_{\text {iso }}(\mathrm{H})$ constrained to be 1.2 times $U_{\text {eq }}$ for the atom it is bonded to.

\section{S3. Results and discussion}

The asymmetric unit comprises one molecule of $\mathrm{C}_{12} \mathrm{H}_{7} \mathrm{~N}_{3} \mathrm{O}_{2} \mathrm{~S}$ (Fig. 1). The phenylthiazolopyridine ring system is flat with a maximum deviation of 0.072 (1) $\AA$ from the least squares plane. The nitro group is twisted from this plane by only $7.6(2)^{\circ}$. In the crystal, extensive $\pi-\pi$ overlap occurs between pairs of inversion related molecules with a phenyl to thiazolopyridine centroid distance of 3.47 (2) $\AA$ (Fig. 2). 


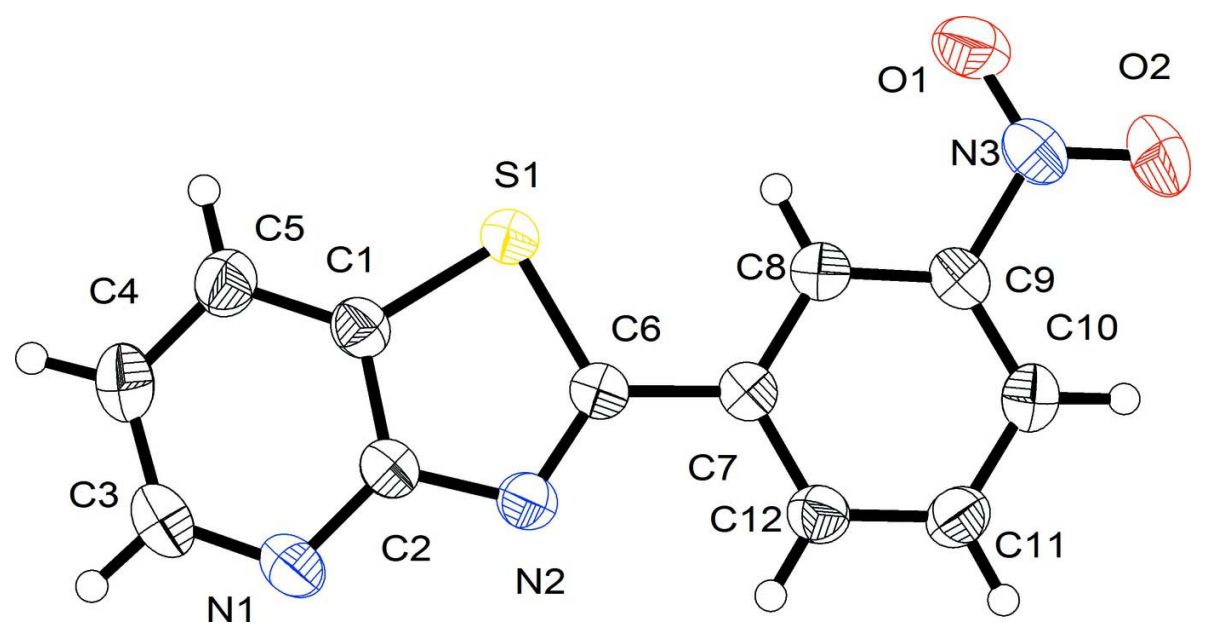

Figure 1

The asymmetric unit of $\mathrm{C}_{12} \mathrm{H}_{7} \mathrm{~N}_{3} \mathrm{O}_{2} \mathrm{~S}$ with $50 \%$ probability displacement ellipsoids for nonhydrogen atoms.

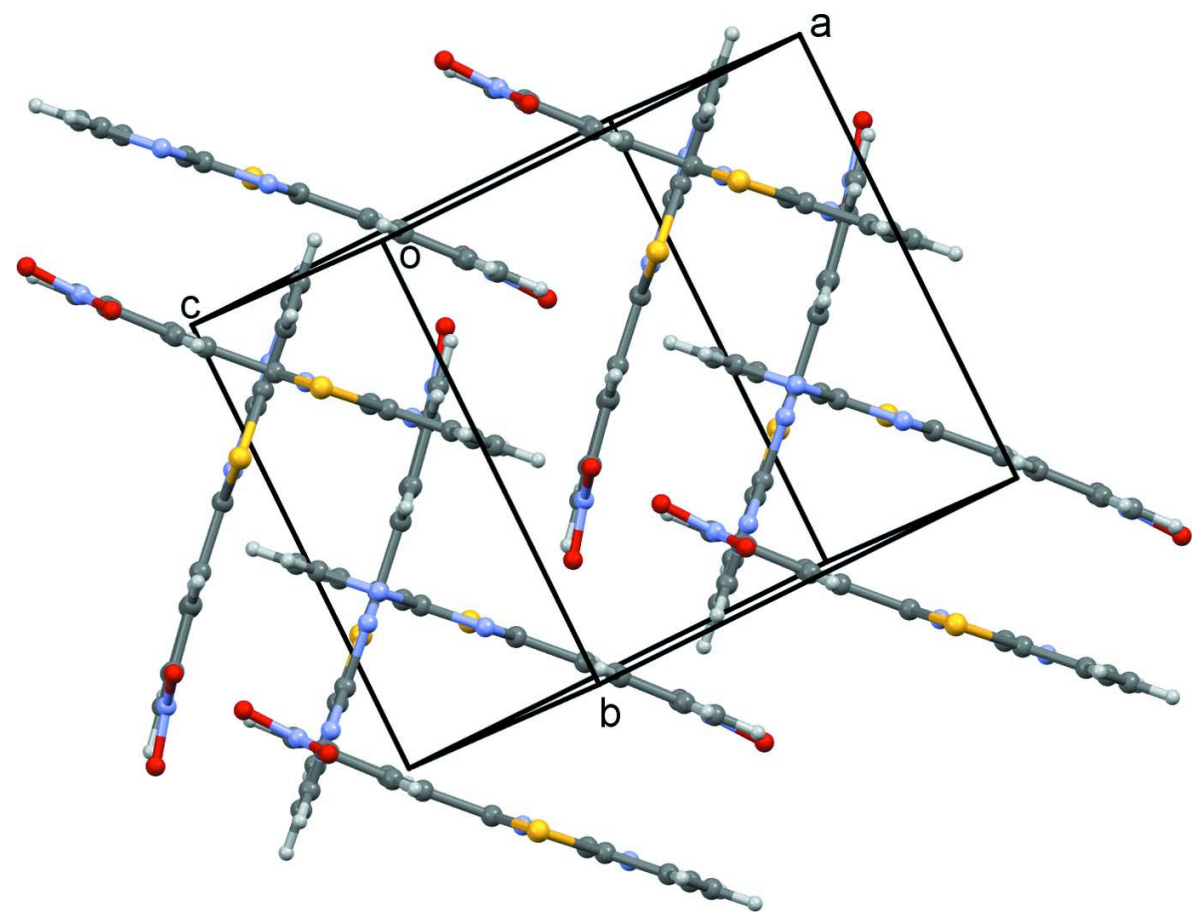

Figure 2

A segment of the crystal structure with $\mathrm{H}$ atoms omitted for clarity.

2-(3-Nitrophenyl)-1,3-thiazolo[4,5-b]pyridine

Crystal data

$\mathrm{C}_{12} \mathrm{H}_{7} \mathrm{~N}_{3} \mathrm{O}_{2} \mathrm{~S}$

$M_{r}=257.27$

Monoclinic, $P 2_{1} / c$

$a=9.5596(2) \AA$

$b=9.8733(2) \AA$

$c=11.5606(3) \AA$

$\beta=98.122(2)^{\circ}$

$V=1080.20(4) \AA^{3}$
$Z=4$
$F(000)=528$
$D_{\mathrm{x}}=1.582 \mathrm{Mg} \mathrm{m}^{-3}$

$\mathrm{Cu} K \alpha$ radiation, $\lambda=1.54184 \AA$

Cell parameters from 2610 reflections

$\theta=5.9-73.8^{\circ}$ 
$\mu=2.66 \mathrm{~mm}^{-1}$

$T=296 \mathrm{~K}$

\section{Data collection}

Agilent SuperNova Dual Source diffractometer with an Atlas detector

$\omega$ scans

Absorption correction: gaussian

(CrysAlis PRO; Agilent, 2014)

$T_{\min }=0.883, T_{\max }=0.986$

4063 measured reflections

\section{Refinement}

Refinement on $F^{2}$

Least-squares matrix: full

$R\left[F^{2}>2 \sigma\left(F^{2}\right)\right]=0.031$

$w R\left(F^{2}\right)=0.086$

$S=1.06$

2104 reflections

163 parameters

0 restraints
Plate, colourless

$0.36 \times 0.24 \times 0.03 \mathrm{~mm}$

2104 independent reflections

1930 reflections with $I>2 \sigma(I)$

$R_{\text {int }}=0.016$

$\theta_{\max }=73.8^{\circ}, \theta_{\min }=5.9^{\circ}$

$h=-6 \rightarrow 11$

$k=-12 \rightarrow 10$

$l=-13 \rightarrow 14$

Hydrogen site location: inferred from neighbouring sites

$\mathrm{H}$-atom parameters constrained

$w=1 /\left[\sigma^{2}\left(F_{0}^{2}\right)+(0.048 P)^{2}+0.2004 P\right]$

where $P=\left(F_{\mathrm{o}}^{2}+2 F_{\mathrm{c}}^{2}\right) / 3$

$(\Delta / \sigma)_{\max }=0.001$

$\Delta \rho_{\max }=0.20$ e $\AA^{-3}$

$\Delta \rho_{\min }=-0.27$ e $\AA^{-3}$

Special details

Experimental. Absorption correction: CrysAlisPro, Agilent Technologies, Version 1.171.37.33 (release 27-03-2014 CrysAlis171 .NET) (compiled Mar 27 2014,17:12:48) Numerical absorption correction based on gaussian integration over a multifaceted crystal model Empirical absorption correction using spherical harmonics, implemented in SCALE3 ABSPACK scaling algorithm.

Geometry. All e.s.d.'s (except the e.s.d. in the dihedral angle between two l.s. planes) are estimated using the full covariance matrix. The cell e.s.d.'s are taken into account individually in the estimation of e.s.d.'s in distances, angles and torsion angles; correlations between e.s.d.'s in cell parameters are only used when they are defined by crystal symmetry. An approximate (isotropic) treatment of cell e.s.d.'s is used for estimating e.s.d.'s involving 1.s. planes.

Fractional atomic coordinates and isotropic or equivalent isotropic displacement parameters $\left(\AA^{2}\right)$

\begin{tabular}{lllll}
\hline & $x$ & $y$ & $z$ & $U_{\text {iso }} * / U_{\text {eq }}$ \\
\hline C1 & $1.03467(14)$ & $0.31902(15)$ & $0.40820(12)$ & $0.0359(3)$ \\
C2 & $1.05966(14)$ & $0.29483(14)$ & $0.52938(12)$ & $0.0346(3)$ \\
C3 & $1.23544(16)$ & $0.44974(17)$ & $0.55180(15)$ & $0.0466(4)$ \\
H3 & 1.3056 & 0.4963 & 0.5999 & $0.056^{*}$ \\
C4 & $1.21719(17)$ & $0.48032(17)$ & $0.43313(15)$ & $0.0476(4)$ \\
H4 & 1.2737 & 0.5453 & 0.4044 & $0.057^{*}$ \\
C5 & $1.11469(16)$ & $0.41357(17)$ & $0.35833(14)$ & $0.0446(3)$ \\
H5 & 1.1001 & 0.4312 & 0.2785 & $0.054^{*}$ \\
C6 & $0.88486(14)$ & $0.15122(14)$ & $0.48354(11)$ & $0.0327(3)$ \\
C7 & $0.78177(13)$ & $0.04398(14)$ & $0.49734(11)$ & $0.0323(3)$ \\
C8 & $0.69113(14)$ & $-0.00588(14)$ & $0.40184(12)$ & $0.0338(3)$ \\
H8 & 0.6911 & 0.0309 & 0.3278 & $0.041^{*}$ \\
C9 & $0.60130(13)$ & $-0.11128(14)$ & $0.41963(12)$ & $0.0342(3)$ \\
C10 & $0.59533(15)$ & $-0.16807(15)$ & $0.52740(13)$ & $0.0392(3)$ \\
H10 & 0.5333 & -0.2386 & 0.5365 & $0.047^{*}$ \\
C11 & $0.68472(16)$ & $-0.11691(16)$ & $0.62203(13)$ & $0.0419(3)$
\end{tabular}




$\begin{array}{lllll}\text { H11 } & 0.6827 & -0.1532 & 0.6960 & 0.050^{*} \\ \text { C12 } & 0.77673(15) & -0.01253(16) & 0.60750(12) & 0.0381(3) \\ \text { H12 } & 0.8362 & 0.0206 & 0.6719 & 0.046^{*} \\ \text { N1 } & 1.16011(14) & 0.35893(14) & 0.60175(11) & 0.0442(3) \\ \text { N2 } & 0.97356(13) & 0.19809(13) & 0.56961(10) & 0.0367(3) \\ \text { N3 } & 0.50993(13) & -0.16657(14) & 0.31766(11) & 0.0420(3) \\ \text { O1 } & 0.51160(13) & -0.11243(14) & 0.22310(10) & 0.0563(3) \\ \text { O2 } & 0.43804(16) & -0.26586(16) & 0.33152(13) & 0.0698(4) \\ \text { S1 } & 0.89807(4) & 0.21799(4) & 0.34482(3) & 0.03972(13)\end{array}$

Atomic displacement parameters $\left(\AA^{2}\right)$

\begin{tabular}{lllllll}
\hline & $U^{11}$ & $U^{22}$ & $U^{33}$ & $U^{12}$ & $U^{13}$ & $U^{23}$ \\
\hline C1 & $0.0339(7)$ & $0.0341(7)$ & $0.0386(7)$ & $0.0002(5)$ & $0.0009(5)$ & $-0.0009(6)$ \\
C2 & $0.0322(7)$ & $0.0348(7)$ & $0.0361(7)$ & $0.0004(5)$ & $0.0023(5)$ & $-0.0029(5)$ \\
C3 & $0.0387(7)$ & $0.0429(8)$ & $0.0565(9)$ & $-0.0067(6)$ & $0.0004(6)$ & $-0.0100(7)$ \\
C4 & $0.0423(8)$ & $0.0379(8)$ & $0.0627(10)$ & $-0.0072(6)$ & $0.0075(7)$ & $0.0012(7)$ \\
C5 & $0.0445(8)$ & $0.0431(8)$ & $0.0458(8)$ & $-0.0041(6)$ & $0.0048(6)$ & $0.0068(6)$ \\
C6 & $0.0318(6)$ & $0.0337(7)$ & $0.0322(6)$ & $0.0021(5)$ & $0.0037(5)$ & $-0.0016(5)$ \\
C7 & $0.0293(6)$ & $0.0323(6)$ & $0.0355(7)$ & $0.0035(5)$ & $0.0049(5)$ & $-0.0017(5)$ \\
C8 & $0.0316(6)$ & $0.0351(7)$ & $0.0346(6)$ & $0.0035(5)$ & $0.0047(5)$ & $-0.0004(5)$ \\
C9 & $0.0279(6)$ & $0.0342(7)$ & $0.0398(7)$ & $0.0040(5)$ & $0.0024(5)$ & $-0.0050(5)$ \\
C10 & $0.0353(7)$ & $0.0349(7)$ & $0.0479(8)$ & $-0.0002(6)$ & $0.0080(6)$ & $0.0028(6)$ \\
C11 & $0.0436(8)$ & $0.0443(8)$ & $0.0380(7)$ & $0.0000(6)$ & $0.0060(6)$ & $0.0068(6)$ \\
C12 & $0.0362(7)$ & $0.0417(8)$ & $0.0355(7)$ & $0.0001(6)$ & $0.0021(5)$ & $-0.0002(6)$ \\
N1 & $0.0410(6)$ & $0.0476(7)$ & $0.0420(7)$ & $-0.0059(5)$ & $-0.0012(5)$ & $-0.0079(6)$ \\
N2 & $0.0358(6)$ & $0.0401(6)$ & $0.0335(6)$ & $-0.0017(5)$ & $0.0029(5)$ & $-0.0017(5)$ \\
N3 & $0.0352(6)$ & $0.0425(7)$ & $0.0467(7)$ & $0.0013(5)$ & $0.0006(5)$ & $-0.0082(6)$ \\
O1 & $0.0582(7)$ & $0.0652(8)$ & $0.0421(6)$ & $-0.0030(6)$ & $-0.0052(5)$ & $-0.0054(6)$ \\
O2 & $0.0684(8)$ & $0.0638(9)$ & $0.0724(9)$ & $-0.0315(7)$ & $-0.0067(7)$ & $-0.0050(7)$ \\
S1 & $0.0419(2)$ & $0.0429(2)$ & $0.0323(2)$ & $-0.00887(14)$ & $-0.00199(14)$ & $0.00294(13)$ \\
& & & & & &
\end{tabular}

Geometric parameters $\left(\hat{A},{ }^{\circ}\right)$

\begin{tabular}{llll}
\hline $\mathrm{C} 1-\mathrm{C} 5$ & $1.383(2)$ & $\mathrm{C} 7-\mathrm{C} 8$ & $1.3935(19)$ \\
$\mathrm{C} 1-\mathrm{C} 2$ & $1.408(2)$ & $\mathrm{C} 7-\mathrm{C} 12$ & $1.3974(19)$ \\
$\mathrm{C} 1-\mathrm{S} 1$ & $1.7224(14)$ & $\mathrm{C} 8-\mathrm{C} 9$ & $1.383(2)$ \\
$\mathrm{C} 2-\mathrm{N} 1$ & $1.3400(19)$ & $\mathrm{C} 8-\mathrm{H} 8$ & 0.9300 \\
$\mathrm{C} 2-\mathrm{N} 2$ & $1.3836(19)$ & $\mathrm{C} 9-\mathrm{C} 10$ & $1.375(2)$ \\
$\mathrm{C} 3-\mathrm{N} 1$ & $1.332(2)$ & $\mathrm{C} 9-\mathrm{N} 3$ & $1.4696(18)$ \\
$\mathrm{C} 3-\mathrm{C} 4$ & $1.391(2)$ & $\mathrm{C} 10-\mathrm{C} 11$ & $1.385(2)$ \\
$\mathrm{C} 3-\mathrm{H} 3$ & 0.9300 & $\mathrm{C} 10-\mathrm{H} 10$ & 0.9300 \\
$\mathrm{C} 4-\mathrm{C} 5$ & $1.379(2)$ & $\mathrm{C} 11-\mathrm{C} 12$ & $1.380(2)$ \\
$\mathrm{C} 4-\mathrm{H} 4$ & 0.9300 & $\mathrm{C} 11-\mathrm{H} 11$ & 0.9300 \\
$\mathrm{C} 5-\mathrm{H} 5$ & 0.9300 & $\mathrm{C} 12-\mathrm{H} 12$ & 1.9300 \\
$\mathrm{C} 6-\mathrm{N} 2$ & $1.2980(18)$ & $\mathrm{N} 3-\mathrm{O} 1$ & $1.221(2)$ \\
$\mathrm{C} 6-\mathrm{C} 7$ & $1.4705(19)$ & $\mathrm{N} 3-\mathrm{O} 2$ & \\
$\mathrm{C} 6-\mathrm{S} 1$ & $1.7548(14)$ & &
\end{tabular}




$\mathrm{C} 5-\mathrm{C} 1-\mathrm{C} 2$
$\mathrm{C} 5-\mathrm{C} 1-\mathrm{S} 1$
$\mathrm{C} 2-\mathrm{C} 1-\mathrm{S} 1$
$\mathrm{~N} 1-\mathrm{C} 2-\mathrm{N} 2$
$\mathrm{~N} 1-\mathrm{C} 2-\mathrm{C} 1$
$\mathrm{~N} 2-\mathrm{C} 2-\mathrm{C} 1$
$\mathrm{~N} 1-\mathrm{C} 3-\mathrm{C} 4$
$\mathrm{~N} 1-\mathrm{C} 3-\mathrm{H} 3$
$\mathrm{C} 4-\mathrm{C} 3-\mathrm{H} 3$
$\mathrm{C} 5-\mathrm{C} 4-\mathrm{C} 3$
$\mathrm{C} 5-\mathrm{C} 4-\mathrm{H} 4$
$\mathrm{C} 3-\mathrm{C} 4-\mathrm{H} 4$
$\mathrm{C} 4-\mathrm{C} 5-\mathrm{C} 1$
$\mathrm{C} 4-\mathrm{C} 5-\mathrm{H} 5$
$\mathrm{C} 1-\mathrm{C} 5-\mathrm{H} 5$
$\mathrm{~N} 2-\mathrm{C} 6-\mathrm{C} 7$
$\mathrm{~N} 2-\mathrm{C} 6-\mathrm{S} 1$
$\mathrm{C} 7-\mathrm{C} 6-\mathrm{S} 1$
$\mathrm{C} 8-\mathrm{C} 7-\mathrm{C} 12$
$\mathrm{C} 8-\mathrm{C} 7-\mathrm{C} 6$
$\mathrm{C} 12-\mathrm{C} 7-\mathrm{C} 6$

$120.20(13)$

$130.10(12)$

$109.69(11)$

$121.61(13)$

$123.16(14)$

$115.23(12)$

$124.99(14)$

117.5

117.5

$119.56(15)$

120.2

120.2

116.54 (14)

121.7

121.7

$123.28(12)$

$116.30(11)$

$120.37(10)$

$118.91(13)$

$121.31(12)$

$119.76(12)$

$\mathrm{C} 9-\mathrm{C} 8-\mathrm{C} 7$
$\mathrm{C} 9-\mathrm{C} 8-\mathrm{H} 8$
$\mathrm{C} 7-\mathrm{C} 8-\mathrm{H} 8$
$\mathrm{C} 10-\mathrm{C} 9-\mathrm{C} 8$
$\mathrm{C} 10-\mathrm{C} 9-\mathrm{N} 3$
$\mathrm{C} 8-\mathrm{C} 9-\mathrm{N} 3$
$\mathrm{C} 9-\mathrm{C} 10-\mathrm{C} 11$
$\mathrm{C} 9-\mathrm{C} 10-\mathrm{H} 10$
$\mathrm{C} 11-\mathrm{C} 10-\mathrm{H} 10$
$\mathrm{C} 12-\mathrm{C} 11-\mathrm{C} 10$
$\mathrm{C} 12-\mathrm{C} 11-\mathrm{H} 11$
$\mathrm{C} 10-\mathrm{C} 11-\mathrm{H} 11$
$\mathrm{C} 11-\mathrm{C} 12-\mathrm{C} 7$
$\mathrm{C} 11-\mathrm{C} 12-\mathrm{H} 12$
$\mathrm{C} 7-\mathrm{C} 12-\mathrm{H} 12$
$\mathrm{C} 3-\mathrm{N} 1-\mathrm{C} 2$
$\mathrm{C} 6-\mathrm{N} 2-\mathrm{C} 2$
$\mathrm{O} 1-\mathrm{N} 3-\mathrm{O} 2$
$\mathrm{O} 1-\mathrm{N} 3-\mathrm{C} 9$
$\mathrm{O} 2-\mathrm{N} 3-\mathrm{C} 9$
$\mathrm{C} 1-\mathrm{S} 1-\mathrm{C} 6$

$118.53(13)$

120.7

120.7

$123.22(13)$

$118.65(13)$

$118.12(13)$

$117.85(13)$

121.1

121.1

$120.58(14)$

119.7

119.7

$120.90(13)$

119.5

119.5

$115.53(14)$

$110.10(12)$

123.28 (14)

$118.36(13)$

$118.35(13)$

88.68 (7) 